Quim. Nova, Vol. 34, No. 6, 940-944, 2011

\title{
DETERMINACIÓN SIMULTÁNEA DE Cr, Ni Y V EN ORINA MEDIANTE ET AAS
}

\author{
Maria A. Álvarez* y Jeymi Hermida \\ Centro de Química Analítica, Escuela de Química, Facultad de Ciencias, Universidad Central de Venezuela, Caracas, 1041-A, \\ Venezuela
}

Recebido em 27/5/10; aceito em 20/1/11; publicado na web em 29/3/11

\begin{abstract}
SIMULTANEOUS DETERMINATION OF Cr, Ni AND V IN URINE BY ET AAS. A procedure for the simultaneous determination of $\mathrm{Cr}, \mathrm{Ni}$, and $\mathrm{V}$ in urine by electrothermal atomic absorption spectrometry (ET AAS) was optimized by factorial design, and performed at a pyrolysis and atomization temperatures of 1300 and $2500{ }^{\circ} \mathrm{C}$, respectively, using $15 \mu \mathrm{g} \mathrm{de} \mathrm{Mg}\left(\mathrm{NO}_{3}\right)_{2}$ as chemical modifier. Characteristics mass of 14,6 and $220 \mathrm{\rho g}$ and detection limits of the method of $0.07,0.38$ and $0.75 \mu \mathrm{g} \mathrm{L}^{-1}$ were obtained for $\mathrm{Cr}, \mathrm{Ni}$ and $\mathrm{V}$ respectively. The methodology was validated using a Liphochek Urine Metals Control sample (Bio-Rad) ( $\mathrm{P}=0.05)$. The methodology was applied to samples of voluntary Venezuelan people, not environmentally exposed to specific emissions, and results ranging from < LOD-1.1 and 1.3-3.3 $\mu \mathrm{g} \mathrm{L}^{-1}$ was observed for $\mathrm{Cr}$ and $\mathrm{V}$, respectively, and not detectable levels for $\mathrm{Ni}$.
\end{abstract}

Keywords: simultaneous analysis; ET AAS; metals in urine.

\section{INTRODUCCIÓN}

El cromo, el níquel y el vanadio forman parte de numerosas aleaciones metálicas, ${ }^{1,2}$ y pueden encontrarse en el aire de ambientes industriales asociados a actividades metalúrgicas, de refinación de minerales, producción de cemento y en plantas de energía que emplean combustibles fósiles. ${ }^{3}$ De manera particular, la fuente más común de $\mathrm{Cr}$ en el ambiente se origina por los desechos de las industrias de cromado y electrodeposición, ${ }^{4,5}$ de textiles y pieles. ${ }^{6}$

Los efectos nocivos del exceso ( $\mathrm{Cr}$, Ni y V) o falta (Cr y V) de estos metales sobre la salud humana son conocidos, ${ }^{3}$ y su cuantificación en fluidos biológicos es importante, especialmente en el monitoreo de deficiencias nutricionales y exposición ambiental. ${ }^{4}$ En el caso de la exposición ambiental, la evaluación de riesgo es realizada usualmente mediante la determinación de los metales presentes en los fluidos biológicos, entre los cuales, la orina es empleada ampliamente debido a que es una muestra fácilmente disponible y un indicador útil de la exposición a metales tóxicos..$^{3-7}$

La concentración de cromo, níquel y vanadio en la orina se encuentra en niveles traza, por lo que su determinación requiere el empleo de técnicas analíticas muy sensibles como la espectrometría de masas con plasma acoplado inductivamente (ICP-MS) y la espectrometría de absorción atómica con atomización electrotérmica (ET AAS). ICP-MS es una técnica sofisticada y costosa que, además de su elevada sensibilidad y capacidad de análisis multielemental, puede presentar interferencias por iones poliatómicos que afectan, entre otras especies, la determinación de ${ }^{52} \mathrm{Cr}$ y ${ }^{51} \mathrm{~V}$ cuando se emplean equipos de baja resolución o sin celda de reacción..$^{8} 10$ Por su parte, ET AAS es una técnica que proporciona la sensibilidad requerida para este tipo de análisis, y su empleo se ve favorecido ya que es menos costosa, muchas veces el pre-tratamiento es más sensible, y las interferencias que presenta pueden solucionarse, generalmente, empleando modificadores químicos, apropiados programas de calentamiento y sistemas de corrección de fondo apropiados. ${ }^{3}$

La aplicación de ET AAS en el análisis unielemental en mues-

*e-mail: maria.alvarez@ciens.ucv.ve tras de orina ha sido ampliamente señalada, específicamente en la determinación de $\mathrm{Cr}^{4-5,11-15}$ y Ni. ${ }^{7,14}$ Asimismo, se ha señalado el análisis multielemental en orina mediante ET AAS, en particular la determinación de cromo, ${ }^{16,17}$ y niquel, ${ }^{18,19}$ y otros elementos, ${ }^{17,18,20}$ así como en otros tipos de muestras como particulado ambiental, ${ }^{21}$ aleaciones de aluminio, ${ }^{22}$ aguas y materiales geológicos, ${ }^{23}$ miel,,${ }^{24} \mathrm{y}$ vino, ${ }^{25}$ entre otras. Hasta donde se conoce, la determinación de $\mathrm{V}$ en orina mediante ET AAS no se ha señalado, empleándose en su lugar ICP-MS con espectrómetros de alta resolución (HR-ICP-MS), ${ }^{10,26}$ con celdas de reacción dinámica, ${ }^{8}$ y octopolos con celda de colisión, ${ }^{9}$ así como la espectrometría de emisión atómica con plasma acoplado inductivamente (ICP OES) aplicando procedimientos previos de separación y preconcentración del elemento, para evitar las interferencias espectrales así como alcanzar los límites de detección requeridos. ${ }^{27}$

La determinación simultánea por ET AAS es posible y deseable en muchos casos, por la ventaja de economía de tiempo de análisis que esta técnica ofrece. Para esto, es necesario buscar un programa de temperatura y un modificador químico que sean adecuados para los elementos de interés. A pesar de que no hay más proveedores de equipos de absorción atómica capaces de realizar análisis multielementales, la introducción de la espectrometría de absorción atómica de fuente continúa y alta resolución es seguramente un paso facilitador para el desarrollo de procedimientos para análisis multielementales por ET AAS, que gana así nuevo interés.

Por otra parte, es importante destacar la importancia del tratamiento de la muestra de orina para su análisis mediante ET AAS, ya que, la presencia de elevadas cantidades de sales disueltas, así como partículas sólidas y materia orgánica en suspensión, promueven importantes interferencias de matriz para los analitos volátiles y señales de fondo elevadas. Para evitar estas interferencias, la muestra usualmente es digerida, ${ }^{7,14}$ o diluida. ${ }^{11,16-18}$ Sin embargo, se prefiere la introducción directa de la muestra, ${ }^{4-5,13,15,19}$ lo cual requiere una cuidadosa selección de las condiciones de análisis, pero minimiza los riesgos de contaminación de la muestra y permite mejorar el tiempo de análisis y el límite de detección del método. En este sentido, se han empleado procedimientos de preconcentración para realizar estudios de especiación en orina, mejorando al mismo tiempo los límites de 
detección, ${ }^{12}$ donde los procedimientos tienden, recientemente, a realizarse en línea. ${ }^{28}$

Este trabajo se enfocó en la optimización de una metodología sencilla para la determinación simultánea de $\mathrm{Cr}$, Ni y $\mathrm{V}$ en orina mediante ET AAS, dada la potencial toxicidad que representan dichos elementos para el ser humano y su presencia conjunta en algunas muestras y ambientes laborales. ${ }^{1-3}$

\section{PARTE EXPERIMENTAL}

\section{Equipos}

Se empleó un espectrómetro de absorción atómica con horno de grafito marca Perkin Elmer, modelo SIMAA 6000, el cual permite la determinación simultánea de hasta seis elementos. Este equipo posee un sistema de corrección de fondo basado en el efecto Zeeman longitudinal y un automuestreador AS-72 y opera con atomizadores calentados transversalmente (THGA). En este trabajo se emplearon lámparas de cátodo hueco de $\mathrm{Cr}(357,9 \mathrm{~nm}), \mathrm{Ni}(232,0 \mathrm{~nm})$ y V $(318,4$ nm) marca Perkin Elmer, modelo Lumina.

\section{Reactivos}

Se emplearon soluciones estándar acuosas High Purity Standards, marca Perkin Elmer, de $1000 \mu \mathrm{g} \mathrm{L}^{-1}$ de Cr, Ni y V; solución estándar de $1000 \mu \mathrm{g} \mathrm{L}{ }^{-1}$ de $\mathrm{Mg}\left(\mathrm{NO}_{3}\right)_{2}$ marca Fluka como modificador químico; Tritón X-100 doble destilado marca Packard Instrument Company, USA; $\mathrm{HNO}_{3}$ para trazas $69-71 \%$ marca J.T. Baker; y agua desionizada grado $18 \mathrm{M}$ ohm $\mathrm{cm}$.

\section{Procedimiento}

\section{Tratamiento de la muestra de orina}

La muestra de orina fue filtrada con un filtro desechable de jeringa (Dismic-25cs, $<45 \mu \mathrm{m}$ ) para eliminar los sólidos suspendidos. Posteriormente, se le agregó $\mathrm{HNO}_{3}$ concentrado y Triton X-100 al $10 \% \mathrm{~m} / \mathrm{v}$, hasta una concentración final en la muestra de $0,1 \% \mathrm{~m} / \mathrm{v}$, en ambos reactivos. Con este procedimiento la dilución de la muestra fue despreciable.

\section{Optimización del programa de calentamiento}

El programa de calentamiento para el análisis simultáneo mediante ET AAS fue optimizado aplicando un diseño factorial $3^{2}$, que consideró dos factores (A: temperatura de pirolisis (Tp); y B: temperatura de atomización (Tat)) y tres niveles para cada factor (A: $1.000,1.150$ y $1.300{ }^{\circ} \mathrm{C}$; y B: $2.200,2.350$ y $2.500{ }^{\circ} \mathrm{C}$ ).

El diseño factorial fue aplicado a una solución estándar acuosa y a la muestra de orina. La solución estándar acuosa, conteniendo concentraciones de $10 \mu \mathrm{g} \mathrm{L}^{-1}$ de $\mathrm{Cr}, 40 \mu \mathrm{g} \mathrm{L} \mathrm{L}^{-1}$ de Ni y $100 \mu \mathrm{g} \mathrm{L}^{-1}$ de $\mathrm{V}$, fue preparada al $0,1 \% \mathrm{~m} / \mathrm{v}$ en $\mathrm{HNO}_{3}$ y Triton $\mathrm{X}-100$. La muestra de orina fue contaminada con una concentración de vanadio similar a la empleada en el estándar acuoso, debido a la baja concentración del elemento observada en dicha muestra. Como modificador químico se empleó $15 \mu \mathrm{g}$ de $\mathrm{Mg}\left(\mathrm{NO}_{3}\right)_{2}$, cantidad recomendada para el cromo en el manual del fabricante. ${ }^{29} \mathrm{El}$ volumen de inyección de la muestra en el atomizador de grafito fue de $20 \mu \mathrm{L}$. El tiempo de integración de la señal atómica fue de $2,5 \mathrm{~s}$ para el $\mathrm{Cr}$ y Ni y de $10 \mathrm{~s}$ para el V, los cuales fueron determinados mediante experiencias preliminares realizadas a las temperaturas de atomización consideradas en el diseño.

Las experiencias requeridas por el diseño se realizaron de manera aleatoria. La señal de respuesta considerada fue la señal de absorbancia atómica integrada, obtenida para cada analito en el modo de análisis simultáneo del equipo. El análisis del diseño factorial fue rea- lizado empleando el programa Statgraphic versión 6.0 para Windows.

\section{Exactitud del método de análisis y determinación de figuras de mérito}

El método optimizado fue validado empleando la muestra control Urine Metals Control Liphochek de Bio-rad Nivel 1, la cual fue regenerada siguiendo las instrucciones del fabricante y tratada para tener una concentración de $0,1 \% \mathrm{~m} / \mathrm{v}$ de $\mathrm{HNO}_{3}$ y Triton X-100. La cuantificación de los analitos se realizó mediante curva de adición de estándar $(\mathrm{n}=5, \mathrm{~m}=3)$, analizando la muestra por triplicado. Adicionalmente, se determinaron las figuras de mérito, masa característica ( $\mathrm{mo}$ ) y límite de detección instrumental (LOD), $(m o=0,0044 / m$ y $L O D=3 \mathrm{sdv} / \mathrm{m}$ donde $m$ es la pendiente analítica de la curva de calibración).

\section{RESULTADOS Y DISCUSIÓN}

\section{Optimización del programa de calentamiento para la determinación simultánea de $\mathrm{Cr}$, Ni y $\mathrm{V}$ en orina por ETAAS}

Las gráficas de Pareto obtenidas en el análisis del diseño factorial aplicado se muestran en la Figura 1, donde se representan los valores estandarizados de los efectos estudiados. El signo de los efectos, indica cómo se modifica la señal analítica cuando se cambia el nivel del factor a un nivel superior. Así, el signo positivo y negativo de los valores estandarizados de los efectos, indican que al aumentar el nivel del factor, la señal de absorbancia atómica integrada aumenta o disminuye, respectivamente.

Los resultados de los efectos principales $(\mathrm{A}=\mathrm{Tp}$ y $\mathrm{B}=$ Tat $)$ mostraron que la matriz de orina no modifica significativamente $(\mathrm{P}=0,05)$ el comportamiento de los analitos durante la etapa de pirolisis, ya que la señal de los analitos en ambas matrices (estándar acuoso y orina) no fue afectada significativamente al variar la temperatura de pirólisis, dentro del intervalo de temperaturas considerado (1.000 a $\left.1.300{ }^{\circ} \mathrm{C}\right)$. Sin embargo, el efecto de la temperatura de atomización sobre las señales de los analitos dependió de la matriz, observándose que el aumento de la temperatura de atomización produce la disminución significativa de la señal atómica del Cr y Ni en la matriz de orina, y el aumento significativo de la señal atómica del $\mathrm{V}$ en ambas matrices (estándar acuoso y orina). Adicionalmente, se obtuvo la interacción significativa $(A B)$ entre los efectos Tat y Tp para el Ni en la matriz orina, así como efectos de segundo orden significativos (BB) para la Tat, para el V y Ni en la matriz de orina y para el Cr y V en la de estándar acuoso, lo cual indica que la matriz de orina afecta el comportamiento de los analitos durante la atomización y que la variación de ambas temperaturas, Tp y Tat, afectaría la señal de los analitos de una manera compleja y diferente en cada matriz.

Las temperaturas óptimas de pirólisis y de atomización para cada elemento en cada matriz, dentro del intervalo de temperaturas considerado en el diseño aplicado, fueron determinadas mediante el paquete estadístico empleando como criterio el máximo valor de absorbancia integrada y se muestran en la Tabla 1. Aunque los resultados mostraron que la Tp no afecta significativamente la señal de los analitos en ambas matrices, las Tp óptimas para el Cr y Ni en la matriz de orina fueron menores que en la de estándar acuoso, y para el V ocurre lo opuesto.

Empleando el mismo paquete estadístico y criterio, se determinaron las temperaturas óptimas de pirólisis y atomización para la determinación simultánea de los elementos en cada matriz, las cuales fueron respectivamente, 1.128 y $2.491{ }^{\circ} \mathrm{C}$ en la matriz de estándar acuoso y de 1.300 y $2.458^{\circ} \mathrm{C}$ en la matriz de orina. La diferencia entre las temperaturas de pirólisis óptimas determinadas para el análisis unielmental y el simultáneo en cada matriz, estándar acuoso y orina, indicaría posibles efectos de la temperatura sobre el comportamiento 
a) Orina
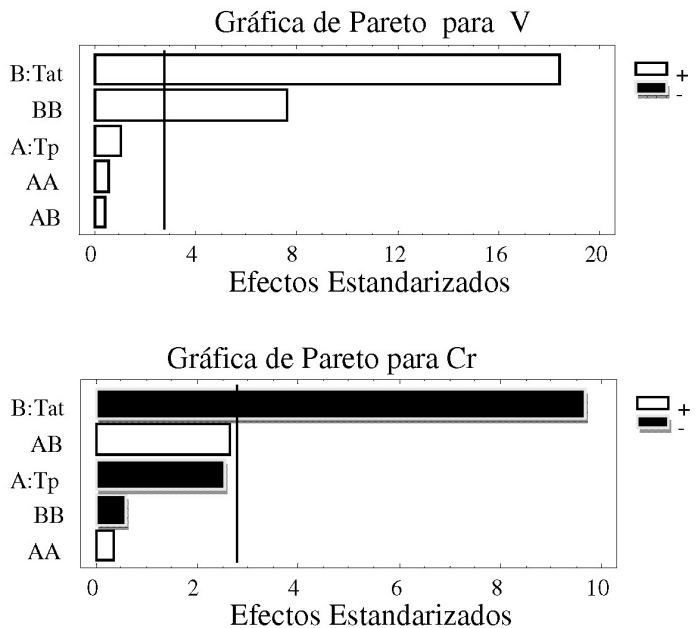

Gráfica de Pareto para $\mathrm{Ni}$



b) Estándar acuoso
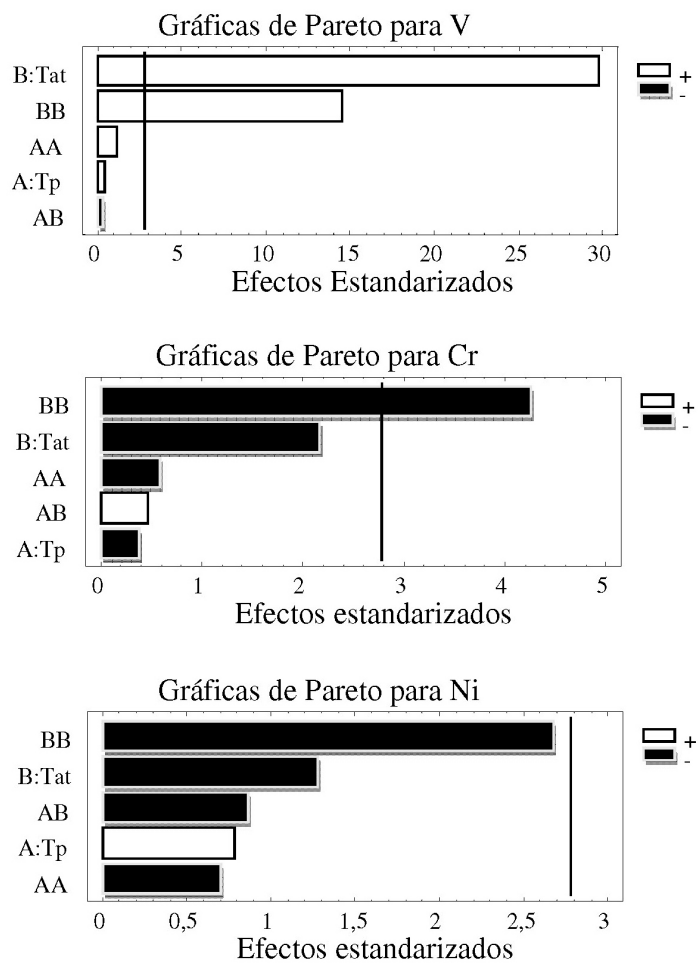

Figura 1. Gráficas de Pareto para los elementos V, Cr y Ni en ambas matrices: a) orina y b) estándar acuoso

Tabla 1. Temperaturas óptimas de pirolisis y atomización* obtenidas en el diseño $3^{2}$

\begin{tabular}{lcccc}
\hline Elemento & \multicolumn{2}{c}{ Estándar acuoso } & \multicolumn{2}{c}{ Orina } \\
& $\mathrm{Tp}$ & Tat & $\mathrm{Tp}$ & Tat \\
\hline $\mathrm{Cr}$ & 1.112 & 2.325 & 1.000 & 2.200 \\
$\mathrm{Ni}$ & 1.216 & 2.320 & 1.000 & 2.459 \\
$\mathrm{~V}$ & 1.000 & 2.500 & 1.300 & 2.500 \\
\hline
\end{tabular}

*Temperaturas en ${ }^{\circ} \mathrm{C}$; Tp: temperatura de pirólisis; Tat: temperatura de atomización.

de los analitos, expresados mediante los efectos principales, las interacciones entre efectos y los efectos de segundo orden determinados en el diseño, los cuales afectarían la señal analítica y en consecuencia, el análisis simultáneo en la matriz orina mediante curva de calibración externa con estándares acuosos.

\section{Análisis simultáneo de $\mathrm{Cr}$, $\mathrm{Ni}$ y $\mathrm{V}$ en orina}

Se hicieron pruebas de calibración aplicando diferentes técnicas de calibración, observándose una disminución del 14, 31 y 11\%, para el $\mathrm{Cr}$, Ni y V, respectivamente, en las pendientes analíticas obtenidas por adición de estándar, respecto a las obtenidas por curva de calibración externa, por lo que el análisis simultáneo se realizó por curva de adición de estándar. Los intervalos de concentración de los elementos empleados en la calibración fueron: 1,3-5 $\mu \mathrm{g} \mathrm{L}^{-1}$ para cromo, 3,8-15 $\mu \mathrm{g} \mathrm{\textrm {L } ^ { - 1 }}$ para níquel y $25-100 \mu \mathrm{g} \mathrm{L}^{-1}$ para vanadio. El análisis se realizó empleando las condiciones compromiso seleccionadas en la optimización, Tp: $1.300^{\circ} \mathrm{C}$ y Tat: $2.500{ }^{\circ} \mathrm{C}$, y $15 \mu \mathrm{g}$ de $\mathrm{Mg}\left(\mathrm{NO}_{3}\right)_{2}$ cómo modificador químico. $\mathrm{La}$ temperatura de pirólisis seleccionada corresponde a la temperatura óptima determinada para el análisis simultáneo de los elementos en la matriz de orina.

\section{Figuras de mérito del método, mo y LOD}

Las figuras de mérito, masa característica y límite de detección del método, obtenidas empleando la metodología optimizada para el análisis simultáneo de Cr, Ni y V en orina se muestran en la Tabla 2. En este trabajo, los límites de detección del método (LODm) son iguales a los LOD instrumentales, ya que la dilución de la muestra fue despreciable. La solución considerada como solución blanco fue una solución acuosa al 0,1\% m/v de $\mathrm{HNO}_{3}$ y Triton X-100.

Tabla 2. Figuras de mérito obtenidas en el análisis simultáneo de Cr, Ni y $\mathrm{V}$ en orina

\begin{tabular}{lcccc}
\hline Elemento & \multicolumn{2}{c}{ Valores obtenidos } & \multicolumn{2}{c}{ Valores reportados* } \\
& $\begin{array}{c}m_{\mathrm{o}} \\
(\rho \mathrm{g})\end{array}$ & $\begin{array}{c}\text { LODm } \\
\left.(\mu \mathrm{g} \mathrm{L})^{-1}\right)\end{array}$ & $\begin{array}{c}m_{\mathrm{o}} \\
(\rho \mathrm{g})\end{array}$ & $\begin{array}{c}\text { LODm } \\
\left(\mu \mathrm{g} \mathrm{L}^{-1}\right)\end{array}$ \\
\hline $\mathrm{Cr}$ & 14 & 0,07 & $7,8^{16}$ & $0,03^{4} ; 0,12^{5} ; 0,44^{11} ;$ \\
$\mathrm{Ni}$ & 55 & 0,38 & $30,3^{18}$ & $0,1^{13} ; 0,15^{15} ; \mathbf{0 . 1 6}^{16}$ \\
$\mathrm{~V}$ & 220 & 0,75 & $51^{30}$ & $0,5^{7} ; \mathbf{0 , 7 0}^{18}$ \\
\hline
\end{tabular}

*Se destacan en negrilla los resultados obtenidos mediante el análisis simultáneo empleando el mismo atomizador PE-SIMAA 6000.

Para el $\mathrm{Cr}$ y el $\mathrm{Ni}$, los valores de LODm obtenidos fueron mejores que los obtenidos por otros autores, tanto en el análisis simultáneo empleando el mismo sistema instrumental (PE-SIMAA $6000),{ }^{16,18}$ como en el análisis unielemental en orina empleando otros instrumentos, ${ }^{5,11,13,15}$ a excepción de los obtenidos para el $\mathrm{Cr}$ por Burguera et al., ${ }^{4}$ quienes realizaron el análisis directo de la muestra mediante curva de calibración externa. Los valores de $m_{\mathrm{o}}$ obtenidos fueron mayores que los señalados por otros autores para el análisis simultáneo empleando el mismo instrumental, ${ }^{16,18,30}$ lo cual se atri- 
buye, además de las diferencias en el programa de calentamiento y tipo de modificador químico empleados y al efecto de la matriz de orina sobre la sensibilidad analítica expresada en la pendiente de la curva de adición estándar aplicada en este trabajo, a diferencia de los trabajos citados donde emplearon curva de calibración externa. Aunque empleando otros espectrómetros, trabajos realizados en el análisis de muestras en suspensión muestran el efecto de la matriz sobre la pendiente analítica, observado mediante los valores de la masa característica, $m_{\mathrm{o}}$ : para el $\mathrm{Cr}$ valores entre $3,5 \rho \mathrm{g}$ y $11,2 \rho \mathrm{g}$ en suspensiones de muestras geológicas a Tp y Tat variables (1.4001.600 y $2.300-2.500{ }^{\circ} \mathrm{C}$, respectivamente),${ }^{31}$ y para el $\mathrm{Ni}$, valores de $26 \rho \mathrm{g}$ en el análisis en suspensiones de aleaciones de aluminio, ${ }^{22} \mathrm{y}$ de 27 gg y $44 \rho g$ en el análisis de suspensiones de muestras de fuel y material del fraccionamiento de crudos empleando Tat de $2.400{ }^{\circ} \mathrm{C} .{ }^{32}$

En el caso del V, se observaron diferencias entre los valores del LODm y $m$ o obtenidos con el método propuesto y los señalados por el fabricante del equipo para el análisis simultáneo del elemento en soluciones acuosas. ${ }^{30}$ Aunque no se encontraron valores de estas figuras de mérito para el análisis unielemental y/o simultáneo en orina, a modo de comparación, pueden señalarse los límites de detección instrumental y masa característica, $0,34 \mu \mathrm{g} \mathrm{L}{ }^{-1}$ y $78 \mu \mathrm{g}$, respectivamente, obtenidos empleando el mismo espectrómetro (PE-SIMAA 6000), a una Tp de $1.700^{\circ} \mathrm{C}$ y Tat de $2.600^{\circ} \mathrm{C}$ en el análisis de suspensiones de cabello humano, ${ }^{33}$ así como otros valores de mo obtenidos empleando otros instrumentos: 20-21 $\rho$ g en el análisis de emulsiones de diesel y productos del fraccionamiento de crudos a una Tat de $2.600{ }^{\circ} \mathrm{C} ;{ }^{32} 72$ $\rho g$ en muestras de petróleo empleando una Tat de $2.700{ }^{\circ} \mathrm{C} ;{ }^{34} 37-288$ $\rho \mathrm{g}$ en muestras de suspensiones de muestras geológicas a una Tat de $2.600{ }^{\circ} \mathrm{C} .{ }^{31} \mathrm{La}$ Tat empleada en el método propuesto $\left(2.500{ }^{\circ} \mathrm{C}\right)$ sería una de las causas de la baja sensibilidad obtenida para el elemento.

En el análisis simultáneo mediante ET AAS, escoger una condición compromiso de análisis puede afectar las figuras de mérito de uno o varios elementos a determinar. Adicionalmente, otro factor que contribuye a la desmejora de la mo y el LOD, son las interferencias espectrales producidas por el solapamiento de líneas de emisión dentro del ancho de banda instrumental del monocromador. ${ }^{35}$ En el caso específico, el espectrómetro PE-SIMAA 6000, detecta interferencias espectrales cuando se emplean conjuntamente lámparas de $\mathrm{Cr}$ y $\mathrm{Ni}$ o de $\mathrm{Ni} \mathrm{y} \mathrm{V}$, por lo que en la determinación simultánea de $\mathrm{Cr}$, Ni y $\mathrm{V}$, el software del equipo modifica automáticamente la frecuencia de modulación de las lámparas y el tiempo de adquisición. Esto conduce a una reducción importante de la intensidad de la radiación que llega al atomizador, pudiendo producir cambios en las figuras de mérito, LOD y $m_{\mathrm{o}},{ }^{35}$ tal como se ha demostrado. ${ }^{22}$ Este efecto sobre las figuras de mérito se vuelve crítico cuando se emplean lámparas poco brillantes, ${ }^{22}$ y en el análisis específico realizado, se considera la causa principal de la baja sensibilidad obtenida para el vanadio.

En este trabajo, el efecto del modo simultáneo de análisis sobre el LOD, fue compensado por la introducción de la muestra de orina prácticamente sin dilución, lo que conduce a valores de los LOD del método mejores, entre dos y seis veces, que los obtenidos en el análisis de la muestra previamente digestada y/o diluida, ${ }^{7,11,16,18}$ debido al factor de dilución empleado.

\section{Exactitud del método de análisis}

La exactitud de la metodología propuesta se determinó analizando la muestra control de orina Urine Metals Control Liphochek Level 1 de Bio-Rad bajo las condiciones óptimas establecidas. Los resultados se muestran en la Tabla 3.

Para el Cr y el Ni no se encontraron diferencias significativas $(\mathrm{P}=$ $0,05)$ entre las concentraciones determinadas y las reportadas en la muestra control. Para el V, cuya concentración no está señalada en la
Tabla 3. Concentraciones obtenidas y reportadas para la muestra Urine Metals Control Liphochek (Bio-rad) Level

\begin{tabular}{lccc}
\hline & \multicolumn{2}{c}{ Reportadas } & Obtenidas $^{1}$ \\
& Intervalo $\left(\mu \mathrm{g} \mathrm{L}^{-1}\right)$ & Promedio $\left(\mu \mathrm{g} \mathrm{L}^{-1}\right)$ & $\mathrm{x} \pm \mathrm{sdv}\left(\mu \mathrm{g} \mathrm{L}^{-1}\right)$ \\
\hline $\mathbf{V}$ & $\mathrm{NR}^{2}$ & $\mathrm{NR}^{2}$ & $104 \pm 3$ \\
$\mathrm{Cr}$ & $1,8-4,1$ & 2,9 & $3,3 \pm 0,4$ \\
$\mathrm{Ni}$ & $6,3-12,3$ & 9,3 & $10 \pm 1$ \\
\hline
\end{tabular}

${ }^{1}$ Concentración promedio \pm desviación estándar para tres réplicas. ${ }^{2} \mathrm{NR}$ : No reportado.

muestra control, se realizó de manera adicional, el análisis mediante curva de calibración externa empleando las mismas condiciones de análisis, puesto que en el estudio de la optimización del programa de calentamiento no se observaron diferencias en el comportamiento del analito en las matrices de orina y acuosa. Mediante esta técnica de calibración se obtuvo una concentración de vanadio en la muestra de $112 \pm 10 \mu \mathrm{g} \mathrm{L}^{-1}$ y un porcentaje de recuperación de $110 \%$, no encontrándose diferencias significativas con la concentración determinada mediante la curva de adición de estándar (prueba $t, \mathrm{P}=0,05$ ).

El método optimizado para el análisis simultáneo fue aplicado a muestras de orina de tres donantes voluntarios saludables, con edades comprendidas entre 19 y 22 años, pertenecientes al laboratorio de investigación que no realizan actividades asociadas a riesgo de contaminación metálica, en las cuales se obtuvieron concentraciones en los intervalos: < LOD-1,1 $\mu \mathrm{g} \mathrm{L}^{-1}$ de Cr y 1,3-3,3 $\mu \mathrm{g} \mathrm{L}^{-1} \mathrm{de} \mathrm{V}$, siendo la concentración de Ni no detectable en estas muestras. Estos valores se encuentran en los intervalos de concentración de referencia señalados para población humana saludable, tales como los determinados para el Cr $\left(0,07-0,88 \mu \mathrm{g} \mathrm{L}^{-1}\right)$ en Italia, ${ }^{3}$ para el Ni $\left(0,59-4,06 \mu \mathrm{g} \mathrm{L}{ }^{-1}\right)$ y V $\left(1,4-10,2 \mu \mathrm{g} \mathrm{L}^{-1}\right)$ en Francia, ${ }^{36}$ y otros valores para el $\mathrm{Cr}(0,24-1,8 \mu \mathrm{g}$ $\left.\mathrm{L}^{-1}\right)$ y el $\mathrm{Ni}\left(2,2-2,7 \mu \mathrm{g} \mathrm{L}^{-1}\right)$, considerados líneas base de acuerdo a datos de 55 paises. ${ }^{37}$

\section{CONCLUSIONES}

La determinación simultánea mediante ET AAS obliga al empleo de condiciones compromiso para el análisis, bajo las cuales la sensibilidad puede ser afectada. Sin embargo, la disminución de la sensibilidad no compromete la capacidad y aplicabilidad de la técnica en el análisis de trazas, permitiendo por el contrario, la mejora del tiempo de análisis y la disminución del consumo de reactivos. El método propuesto provee la ventaja de permitir la determinación simultánea de Cr, Ni y V en orina, sin diluir y tratada de manera simple y rápida, lo cual evita su contaminación durante la manipulación, disminuye el tiempo de análisis y mejora el límite de detección del método. Por otra parte, el análisis mediante adición de estándar, en el presente caso, no conduce a un aumento significativo del tiempo de análisis, ya que el instrumental empleado (PE-SIMAA 6000) realiza el procedimiento de manera automática empleando, prácticamente, el mismo tiempo que emplearía para curva de calibración externa.

Durante el estudio, se observó la acumulación de sales en el atomizador luego de analizar cierto número de muestras, lo cual promueve la irreproducibilidad de la señal. Este inconveniente fue resuelto, incorporando una etapa extra de limpieza al programa de calentamiento y una solución equivalente al blanco de calibración cada diez inyecciones al atomizador.

\section{AGRADECIMIENTOS}

Los autores agradecen a los árbitros su contribución a la mejora del manuscrito, y al Consejo de Desarrollo Científico y Humanístico 
$(\mathrm{CDCH})$ de la Universidad Central de Venezuela por el financiamiento recibido CDCH-PG-03-124301-99.

\section{REFERENCIAS}

1. Nishimura, C.; Komaki, M.; Hwang, S.; Amano, M.; J. Alloys Compd. 2002, 330-332, 902.

2. Yujun, C.; Zhiying, L.; Wenya, Y.; Xinbo, Z.; Minshou, Z.; J. Appl. Electrochem. 2000, 36, 739.

3. Apostoli, P.; J. Chromatogr., B: Anal. Technol. Biomed. Life Sci. 2002, $778,63$.

4. Burguera, J. L.; Burguera, M.; Rondon, C.; Rodriguez, L.; Carrero, P.; Petit de Peña, Y.; Burguera, E.; J. Anal. At. Spectrom. 1999, 14, 821.

5. Sarmiento, A.; Rojas, M.; Agreda, O.; Seijas, D.; Alvarez, M.; Rev. Bras. Toxicol. 2008, 21, 70.

6. Peña, E.: Palacios, M.; Ospina-Alvarez, N.; Algas como indicadores de contaminación, Programa Editorial Universidad del Valle: Cali, 2005.

7. Ching-Jyi, H.; Jin-Lian, T.; Ping-Hua, H.; Shih-Ching, L.; Shinine-Ren, L.; Cherng-Chyi, T.; Talanta 2002, 56, 1109.

8. Nixon, D. E.; Neubauer, K. R.; Eckdahl, S. J.; Butz, J.A.; Burritt, M. F.; Spectrochim. Acta, Part B 2002, 57, 951.

9. Heitland, P.; Köster, H.; J. Anal. At. Spectrom. 2004, 19, 1552.

10. Sarmiento-González, A.; Marchante-Gayón, J. M.; Tejerina-Lobo, J. M.; Paz-Jiménez, J.; Sanz-Medel, A.; Anal. Bioanal. Chem. 2008, 39, 2583.

11. Linces, K.; Leslis, A.; Gonçalves, C.; Aparecida, C.; Bento, J.; Anal. Bional. Chem. 2002, 374, 1301.

12. Siles, M. T.; Vereda, E.; García, A.; Cano, J.; J. Anal. At. Spectrom. 2004, 19, 398.

13. Barbosa Jr., F.; Simião de Souza, S.; Santos Jr., D.; Krug, F.; Microchem. J. 2004, 78,7 .

14. Afridi, H. I.; Kazi, T. G.; Jamali, M. K.; Kazi, G. H.; Arain, M. B.; Jalbani, N.; Shar, G. Q.; Sarfaraz, R. A.; Toxicology and Industrial Health 2006, 22, 381.

15. Tosoroska, N.; Karadjova, I.; Arpadjan, S.; Stafilov, T.; Central European J. Chem. 2007, 5, 230.
16. Oliveira, P. V.; Oliveira, E.; Fresenius J. Anal. Chem. 2001, 371, 901.

17. White, A.; Panayi, A.; At. Spectrosc. 1998, 19, 89.

18. Man-Ching, H.; Yu-Hsiang, S.; Sahang-Da, H.; Talanta 2004, 62, 791.

19. Yu-Hsiang, S.; Shang-Da, H.; Anal. Chim. Acta 2003, 495, 165.

20. Jiménez, Y.; Alvarez, M. A.; Book of Abstracts, Sixth Rio Symposium on Atomic Spectrometry, Concepción-Pucon, Chile, 2000.

21. Pancras, J. P.; Ondov, J. M.; Zeisler, R.; Anal. Chim. Acta 2005, 38, 303.

22. Carrrión, N.; Itriago, A. M.; Alvarez, M. A.; Eljuri, E.; Talanta 2003, 61, 621.

23. Sen Gupta, J. G.; Bouvier, J. L.; Talanta 1995, 42, 269.

24. Ajtony, Z.; Bencs, L.; Haraszi, R.; Szigeti, J.; Szoboszlai, N.; Talanta 2007, 71, 693.

25. Ajtony, Z.; Szoboszlai, N.; Klaudia, S.; Mezei, P.; György, K.; Bencs, L.; Talanta 2008, 76, 627.

26. Alimonti, A.; Petrucci, F.; Krachler, M.; Bocca, B.; Caroli, S.; J. Environ. Monit. 2000, 2, 351.

27. Pacheco, P. H.; Olsina, R. A.; Smichowski, P.; Martinez, L.; Talanta 2008, 74, 593.

28. Burguera, J.; Burguera, M.; Spectrochim. Acta, Part B 2009, 64, 451.

29. Perkin Elmer; Part Number 0993-5220, Publication B3210.20, 1995.

30. Perkin Elmer; BSW AA product department, AB95S81.

31. Felipe-Sotelo, M.; Carlosena, M. A.; Andrade, J. M.; Cal-Prieto, M. J.; Fernández, E.; Prada, D.; Microchem. J. 2005, 81, 217.

32. Silva, M. M.; Damin, I. C. F.; Goreti, M.; Welz, B.; Talanta 2007, 71, 1877.

33. Fernandes, E. G.; Nogueira, A. R. A.; Gomes, N. J.; Nóbrega, J. A.; Talanta 2007, 71, 1118.

34. Brandão, G. P.; de Campos, R. C.; de Castro, E. V.; de Jesus, H. C.; Spectrochim. Acta, Part B 2007, 62, 962.

35. Schlemmer, G.; Raziuk, B.; Analytical graphite furnace atomic absorption spectrometry: a laboratory guide, Birkäuser Verlag: Basel, 1999.

36. Goulle, J. P.; Mathieu, L.; Castermant, J.; Neveu, N.; Bonneau, L.; Lainé, G.; Bouige, D.; Lacroix, C.; Forensic International 2005, 153, 39.

37. Ivengar, V.; Wolttiez, J.; Clin. Chem. 1998, 34, 474. 